\title{
Quadrupolar transfer pathways
}

\author{
Sasa Antonijevic ${ }^{\mathrm{a}, *}$, Geoffrey Bodenhausen ${ }^{\mathrm{a}, \mathrm{b}}$ \\ a Laboratoire de Résonance Magnétique Biomoléculaire, Institut des Sciences et Ingénierie Chimiques, Ecole Polytechnique Fédérale de Lausanne, \\ Batochime, CH-1015 Lausanne, Switzerland \\ b Département de Chimie, associé au CNRS, Ecole Normale Supérieure, 24 rue Lhomond 75231, Paris Cedex 05, France
}

Received 8 December 2005; revised 2 February 2006

Available online 28 February 2006

\begin{abstract}
A set of graphical conventions called quadrupolar transfer pathways is proposed to describe a wide range of experiments designed for the study of quadrupolar nuclei with spin quantum numbers $I=1,3 / 2,2,5 / 2$, etc. These pathways, which inter alea allow one to appreciate the distinction between quadrupolar and Zeeman echoes, represent a generalization of the well-known coherence transfer pathways. Quadrupolar transfer pathways not merely distinguish coherences with different orders $-2 I \leqslant p \leqslant+2 I$, but allow one to follow the fate of coherences associated with single transitions that have the same coherence order $p=m_{I}^{r}-m_{I}^{s}$ but can be distinguished by a satellite order $q=\left(m_{I}^{r}\right)^{2}-\left(m_{I}^{s}\right)^{2}$.
\end{abstract}

(c) 2006 Elsevier Inc. All rights reserved.

Keywords: Coherence transfer pathways; Coherence order; Satellite order; Phase cycling; Quadrupolar echo

\section{Introduction}

Coherence transfer pathways [1-5], have become popular not only to describe a variety of experiments designed for systems containing coupled spins with $I=1 / 2$, but also for methods intended to characterize quadrupolar spins with $I>1 / 2$. Three reasons appear to contribute to the popularity of coherence transfer pathways: (i) they supplement the information conveyed by pulse sequence diagrams, (ii) they allow one to derive appropriate phasecycles or pulsed field gradient schemes to select desired pathways, and (iii) they allow one to define the conditions that must be fulfilled to obtain pure absorptive line-shapes in two-dimensional spectra. However, conventional coherence transfer pathways are not very well adapted to describe experiments designed for quadrupolar spins. In particular, they fail to describe the distinction between Zeeman echoes and quadrupolar echoes [6-10]. This paper

\footnotetext{
* Corresponding author. Fax: +41 216939435.

E-mail address: Sasa.Antonijevic@epfl.ch (S. Antonijevic).
}

describes quadrupolar transfer pathways, an extension of the graphical conventions of "ordinary" coherence transfer pathways. While the latter merely distinguish different coherence orders $p=m_{I}^{r}-m_{I}^{s}$, the new formalism keeps track of coherences that have the same order $p$ but different satellite orders $q=\left(m_{I}^{r}\right)^{2}-\left(m_{I}^{s}\right)^{2}$. The index $q$ can adopt $2 I+1-|p|$ different integer values corresponding to the number of lines in the $p$-quantum spectrum.

\section{Classification of elements of density operators}

Elements of the density operator can be classified according to coherence order $p$. This quantum number describes the effect of a rotation about the quantization axis $z$ on density operator terms $\sigma^{p}$ of coherence order $p$ [11]

$\exp \left\{-\mathrm{i} \phi I_{z}\right\} \sigma^{p} \exp \left\{+\mathrm{i} \phi I_{z}\right\}=\sigma^{p} \exp \{-\mathrm{i} p \phi\}$.

For quadrupolar spins, irreducible tensor operators $T_{\ell, p}$ of rank $\ell$ and coherence order $p$ provide a convenient basis set to expand the density operator [12-14]. The matrix representations of tensor operators for spin $I=1$ are shown in Fig. 1. Tensor operators obey simple rules 


$$
\begin{aligned}
& \text { Tensor Operators } T_{\ell, p}(I=1) \\
& \frac{1}{\sqrt{3}}\left(\begin{array}{lll}
1 & 0 & 0 \\
0 & 1 & 0 \\
0 & 0 & 1
\end{array}\right) \\
& \frac{1}{\sqrt{2}}\left(\begin{array}{lll}
0 & 0 & 0 \\
1 & 0 & 0 \\
0 & 1 & 0
\end{array}\right) \quad \frac{1}{\sqrt{2}}\left(\begin{array}{ccc}
1 & 0 & 0 \\
0 & 0 & 0 \\
0 & 0 & -1
\end{array}\right) \quad \frac{1}{\sqrt{2}}\left(\begin{array}{ccc}
0 & -1 & 0 \\
0 & 0 & -1 \\
0 & 0 & 0
\end{array}\right) \quad \ell=1 \\
& \left(\begin{array}{lll}
0 & 0 & 0 \\
0 & 0 & 0 \\
1 & 0 & 0
\end{array}\right) \frac{1}{\sqrt{2}}\left(\begin{array}{ccc}
0 & 0 & 0 \\
1 & 0 & 0 \\
0 & -1 & 0
\end{array}\right) \quad \frac{1}{\sqrt{6}}\left(\begin{array}{ccc}
1 & 0 & 0 \\
0 & -2 & 0 \\
0 & 0 & 1
\end{array}\right) \quad \frac{1}{\sqrt{2}}\left(\begin{array}{ccc}
0 & -1 & 0 \\
0 & 0 & 1 \\
0 & 0 & 0
\end{array}\right)\left(\begin{array}{lll}
0 & 0 & 1 \\
0 & 0 & 0 \\
0 & 0 & 0
\end{array}\right) \quad \ell=2 \\
& p=\quad-2 \\
& -1 \quad 0 \\
& +1 \\
& +2 \\
& \text { Single-Element Operators } I_{p, q}(I=1) \\
& \left(\begin{array}{lll}
0 & 0 & 0 \\
0 & 0 & 0 \\
0 & 1 & 0
\end{array}\right) \\
& \left(\begin{array}{lll}
0 & 1 & 0 \\
0 & 0 & 0 \\
0 & 0 & 0
\end{array}\right) \\
& q=+1 \\
& \left(\begin{array}{lll}
0 & 0 & 0 \\
0 & 0 & 0 \\
1 & 0 & 0
\end{array}\right) \\
& p=\quad-2 \\
& \left(\begin{array}{lll}
0 & 0 & 0 \\
1 & 0 & 0 \\
0 & 0 & 0
\end{array}\right) \\
& \left(\begin{array}{lll}
0 & 0 & 0 \\
0 & 0 & 1 \\
0 & 0 & 0
\end{array}\right) \\
& \left(\begin{array}{lll}
0 & 0 & 1 \\
0 & 0 & 0 \\
0 & 0 & 0
\end{array}\right) \quad q=0 \\
& -1 \\
& 0 \\
& +1 \\
& \text { Polarisation Operators } I_{p, q}^{r}(I=1)
\end{aligned}
$$

$\left(\begin{array}{lll}1 & 0 & 0 \\ 0 & 0 & 0 \\ 0 & 0 & 0\end{array}\right)$
$I_{0,0}^{+1}=\left(\begin{array}{lll}0 & 0 & 0 \\ 0 & 1 & 0 \\ 0 & 0 & 0\end{array}\right)$
$I_{0,0}^{0}$$\left(\begin{array}{lll}0 & 0 & 0 \\ 0 & 0 & 0 \\ 0 & 0 & 1\end{array}\right)$

Fig. 1. Top: matrix representations of conventional tensor operators $T_{\ell, p}$ with rank $\ell$ and coherence order $p$ in the base $\left(\left|m_{I}=+1\right\rangle,\left|m_{I}=0\right\rangle,\left|m_{I}=-1\right\rangle\right)$ of a spin $I=1$. Middle: single-element operators $I_{p, q}=|r\rangle\langle s|$ defined in this work, with coherence order $p=m_{I}^{r}-m_{I}^{s}$ and satellite order $q=\left(m_{I}^{r}\right)^{2}-\left(m_{I}^{s}\right)^{2}$. Bottom: the set can be completed with $2 I+1$ polarization operators $I_{0,0}^{r}=|r\rangle\langle r|$ that all have $p=q=0$, but can be distinguished by the index $r=m_{I}^{r}$. Generalizations for spins $I>1$ are straightforward.

under non-selective rotations induced by radio-frequency $(\mathrm{RF})$ pulses with $\omega_{\mathrm{RF}} \gg \omega_{\mathrm{Q}}^{\mathrm{PAS}}$ (which is the exception rather than the rule in quadrupolar NMR), since the coefficients are given by Wigner rotation matrix elements [12-14]

$T_{l, p} \stackrel{\beta_{\phi}}{\rightarrow} \sum_{p^{\prime}=-l}^{l} d_{p^{\prime}, p}^{l}(\beta) \exp \left\{-\mathrm{i}\left(p^{\prime}-p\right) \phi\right\} T_{l, p^{\prime}}$

where $\beta$ is the nutation angle of a non-selective RF pulse, and $\phi$ is its phase with respect to the y-axis, so that an $\mathrm{x}$ pulse has a phase $\phi=270^{\circ}$. The reduced Wigner rotation matrix elements $d_{p^{\prime}, p}^{l}(\beta)$ are tabulated in various works of reference [13-15].

To describe experiments intended to characterize quadrupolar spins $(I>1 / 2)$, we advocate the use of single-element operators $I_{(r, s)}$ (Fig. 1). Each transition between two eigenstates $|r\rangle$ and $|s\rangle$ can be associated with two coherences described by the two single-element operators $I_{(r, s)}=|r\rangle\langle s|$ and $I_{(s, r)}=|s\rangle\langle r|[3,16,17]$. These are not Hermitian, in contrast to single transition operators or fictitious spin $1 / 2$ operators. These two coherences have opposite coherence orders $p\left(I_{(r, s)}\right)=m_{I}^{r}-m_{I}^{s}$ and $p\left(I_{(s, r)}\right)=m_{I}^{s}-m_{I}^{r}=-p\left(I_{(r, s)}\right)$.
To obtain pathway diagrams that are tailored for quadrupolar spins, we suggest that the single-element coherences $I_{(r, s)}$ be re-labeled by using the symbol $I_{p, q}$ (Fig. 2), where $p$ is the usual coherence order and $q$ is the satellite order defined by $q=\left(m_{I}^{r}\right)^{2}-\left(m_{I}^{s}\right)^{2}$. This index $q$ allows one to distinguish the $2 I+1-|p|$ coherences associated with each value of $p$. To describe second- and higherorder quadrupole contributions, one may use higher terms such as $q_{(3)}=\left(m_{I}^{r}\right)^{3}-\left(m_{I}^{s}\right)^{3}$, etc. Note that all polarization operators $|r\rangle\langle r|$ have $p=q=0$, so that the satellite order $q$ does not allow one to distinguish them. If necessary, the polarization operators $I_{0,0}^{r}$ can be distinguished by the index $r=m_{I}^{r}$. A single-element operator $I_{p, q}=|r\rangle\langle s|$ should not be confused with an irreducible tensor operator $T_{\ell, p}$. As may be appreciated from the matrices in Fig. 1, the two sets can be readily interconverted by linear combinations.

The interaction between the electric quadrupole moment of a nucleus and the local electric field gradient results in a perturbation of the Zeeman energy levels. The first-order contribution $\omega_{\mathrm{Q}}^{(1)}$ of the quadrupolar interaction to the 
A

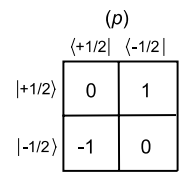

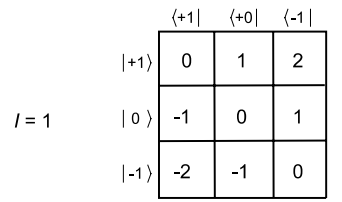

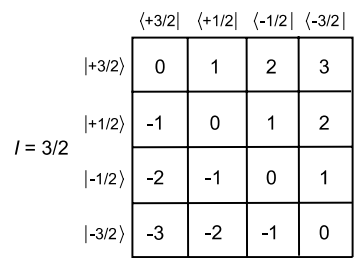

B

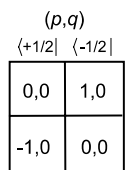

\begin{tabular}{|c|c|c|}
\hline$\langle+1|$ & $\langle+0|$ & $\langle-1|$ \\
\hline 0,0 & $1,+1$ & 2,0 \\
\hline$-1,-1$ & 0,0 & $1,-1$ \\
\hline$-2,0$ & $-1,+1$ & 0,0 \\
\hline
\end{tabular}

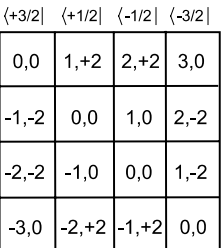

C

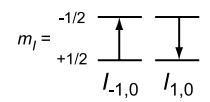

D
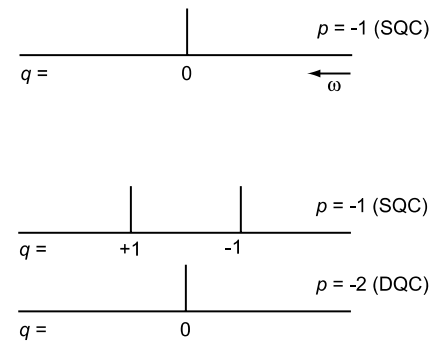

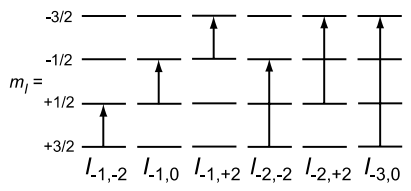
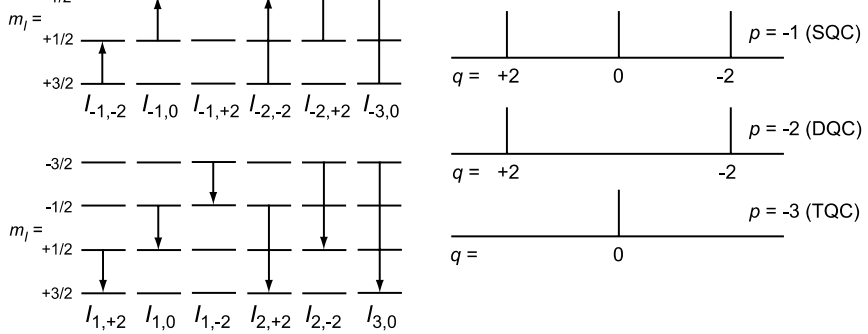

Fig. 2. Elements of matrix representations of density operators for spins $I=1 / 2,1$, and 3/2. (A) The usual classification of a coherent superposition $|r\rangle\langle s|$ between two states $|r\rangle$ and $|s\rangle$ according to the coherence order $p=m_{I}^{r}-m_{I}^{s}$. This classification of the elements does not allow one to distinguish between the $2 I+1-|p|$ coherences with the same order $p$. (B) Proposal for a classification of the elements $I_{p, q}=|r\rangle\langle s|$ distinguished according to the satellite order $q=\left(m_{I}^{r}\right)^{2}-\left(m_{I}^{s}\right)^{2}$. (C) Elements $I_{p, q}=|r\rangle\langle s|$ for spins $I=1 / 2,1$, and 3/2, where a coherent superposition $|r\rangle\langle s|$ between a pair of eigenstates $|r\rangle$ and $|s\rangle$ is described by an arrow $|s\rangle \rightarrow|r\rangle$. (D) Schematic $p$-quantum spectra showing transitions distinguished by their satellite order $q$. The intensities of the individual lines are drawn equal for simplicity. The quadrupolar splittings for $I=1$ and $3 / 2$ correspond to multiples of the first-order quadrupolar interaction $\omega_{\mathrm{Q}}$. Both the gyromagnetic ratio $\gamma$ and the quadrupolar coupling constant $C_{\mathrm{Q}}$ are assumed to be positive.

frequency of a single-element operator $I_{p, q}$, henceforth simply called $\omega_{\mathrm{Q}}$, is given by

$q \omega_{\mathrm{Q}}=q \omega_{\mathrm{Q}}^{\mathrm{PAS}}\left[\frac{3 \cos ^{2} \theta^{\prime}-1}{2}+\frac{\eta_{\mathrm{Q}}}{2} \sin ^{2} \theta^{\prime} \cos 2 \phi^{\prime}\right]$,

where $\theta^{\prime}$ and $\phi^{\prime}$ are the Euler angles describing the transformation from the principal axis system of the quadrupolar tensor to the laboratory frame, $\omega_{\mathrm{Q}}^{\mathrm{PAS}} / 2 \pi=3 C_{\mathrm{Q}} / 4 I(2 I-1)$ is the quadrupolar interaction parameter, $C_{\mathrm{Q}}=e^{2} q Q / h$ the quadrupolar coupling constant, and $\eta_{\mathrm{Q}}$ the asymmetry parameter.

The transformations of $I_{p, q}$ during a free precession interval $t$ under an offset $\Omega$ and a first-order quadrupolar interaction $\omega_{\mathrm{Q}}$ are given by the following rules:

$I_{p, q} \stackrel{\Omega t}{\rightarrow} \exp \{-\mathrm{i} p \Omega t\} I_{p, q}$,

$I_{p, q} \stackrel{\omega_{\mathrm{Q}} t}{\rightarrow} \exp \left\{-\mathrm{i} q \omega_{\mathrm{Q}} t\right\} I_{p, q}$.

Indeed, it is well known that the offset $\Omega$ is 'amplified' for higher-quantum transitions in proportion to the coherence order $p$. The satellite order gives a direct measure of the first-order quadrupolar splittings as illustrated in Fig. 2D. For $I=1$ the SQC doublet $\left(\omega_{\mathrm{Q}},-\omega_{\mathrm{Q}}\right)$ corresponds to $q=(1,-1)$, and the DQC singlet to $q=0$. For $I=3 / 2$ the SQC triplet $\left(2 \omega_{\mathrm{Q}}, 0,-2 \omega_{\mathrm{Q}}\right)$ corresponds to $q=(+2,0,-2)$, the DQC doublet $\left(2 \omega_{\mathrm{Q}},-2 \omega_{\mathrm{Q}}\right)$ to $q=(+2,-2)$, and the TQC singlet to $q=0$. The generalization to spins with $I>3 / 2$ is straightforward. Thus for
$I=5 / 2$ the SQC quintuplet $\left(4 \omega_{\mathrm{Q}}, 2 \omega_{\mathrm{Q}}, 0,-2 \omega_{\mathrm{Q}},-4 \omega_{\mathrm{Q}}\right)$ corresponds to $q=(+4,+2,0,-2,-4)$, the DQC quartet $\left(6 \omega_{\mathrm{Q}}, 2 \omega_{\mathrm{Q}},-2 \omega_{\mathrm{Q}},-6 \omega_{\mathrm{Q}}\right)$ to $q=(+6,+2,-2,-6)$, the TQC triplet $\left(6 \omega_{\mathrm{Q}}, 0,-6 \omega_{\mathrm{Q}}\right)$ to $q=(+6,0,-6)$, the $4 \mathrm{QC}$ doublet $\left(4 \omega_{\mathrm{Q}},-4 \omega_{\mathrm{Q}}\right)$ to $q=(+4,-4)$, and the 5QC singlet to $q=0$.

To form a Zeeman echo, the sign of the coherence order must be changed $\left(p^{\prime}=-p\right)$ between the de- and refocusing intervals of equal length $\tau_{\text {def }}=\tau_{\text {ref }}$, so that the effective offset changes sign $\left(p^{\prime} \Omega=-p \Omega\right)$, thus resulting in a cancellation of the accumulated phase $\phi=p \Omega \tau_{\text {def }}+p^{\prime} \Omega \tau_{\text {ref }}=0$. On the other hand, to form a first order quadrupolar echo, one has to change the sign of the satellite index, so that the accumulated phase vanishes $\phi=q \omega_{\mathrm{Q}} \tau_{\text {def }}+q^{\prime} \omega_{\mathrm{Q}} \tau_{\text {ref }}=0$. In general, coherence transfer echoes $[3,11]$ will occur when both $p$ and $q$ change, i.e., when $\phi=\left(p \Omega+q \omega_{\mathrm{Q}}\right)$ $\tau_{\text {def }}+\left(p^{\prime} \Omega+q^{\prime} \omega_{\mathrm{Q}}\right) \tau_{\text {ref }}=0$ [8]. It is therefore important to keep track of the satellite index $q$ in addition to the coherence order $p$ when drawing coherence transfer pathway diagrams. This, in a nutshell, is the subject of this paper.

Fig. 3 shows the distinction between Zeeman and quadrupolar echoes for spin $I=1$ in terms of "coherence transfer walkways" such as advocated by Kwak and Gan [18]. Such coherence transfer processes can also be drawn on the energy level diagrams. Fig. 4 shows pulse sequences for three basic echo experiments, together with (conventional) coherence transfer pathways (that allow one to keep track of $p$ only) for a spin $I=1$, and (novel) quadrupolar 

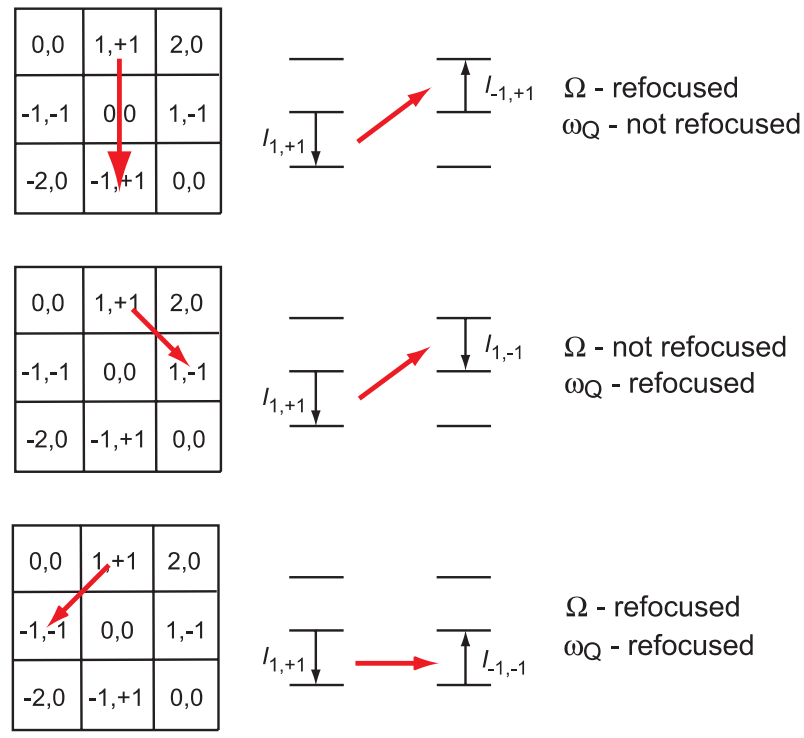

Fig. 3. Coherence transfer processes for spin $I=1$ between singlequantum coherences $I_{p, q}$ of coherence order $p$ and satellite order $q$. Left: drawn on top of the matrix representations of the density operator in the manner of "coherence transfer walkways." Right: in terms of energy level diagrams where the coherences are represented by arrows pointing down or up for $p=+1$ or -1 , respectively. If the sign of $p$ is changed, the Zeeman interaction (offset) is refocused; if the satellite order $q$ changes sign, the first-order quadrupole interaction is refocused.

transfer pathways that show $q$ in addition to $p$. All three pulse sequences feature a defocusing interval $\tau_{\text {def }}$ during which the coherences evolve under shifts and first-order quadrupolar interactions. For the Carr-Purcell echo sequence in Fig. 4A, the $\pi$ pulse results in a change in sign of the coherence order $p$ but not of the satellite order $q$. For spin $I=1$, the single-element operators undergo the following transformations with $\Delta p=p^{\prime}-p$

$I_{p, q} \stackrel{\pi_{\phi}}{\rightarrow} \exp \{-\mathrm{i} \Delta p \phi\} I_{-p, q}$.

Since the evolution under the offset $\Omega$ changes sign, a Zeeman echo will occur after a delay $\tau_{\text {ref, }}$, while the evolution under the first-order quadrupolar interaction $\omega_{\mathrm{Q}}$ keeps the same sign $\left(q^{\prime}=q\right)$, so that no first-order quadrupolar echo can be formed. This subtle distinction is obvious from inspection of the quadrupolar transfer pathways in Fig. 4A.

In order to refocus the first-order quadrupolar interaction it is necessary to change the sign $q^{\prime}=-q$. This can be achieved with the quadrupolar echo sequences of Figs. $4 \mathrm{~B}$ and $\mathrm{C}$ that utilize $\pi / 2$ pulses. For spin $I=1$ one can readily show by converting the $I_{p, q}$ into $T_{\ell, p}$, using Eq. (2), and reconverting $T_{\ell, p^{\prime}}$ back into $I_{p^{\prime}, q^{\prime}}$ that:

$$
\begin{aligned}
I_{1,+1} \stackrel{(\pi / 2)_{\phi}}{\rightarrow} & -\frac{1}{2 \sqrt{2}} \exp \{\mathrm{i} \phi\}\left(I_{0,0}^{+1}-I_{0,0}^{-1}\right) \\
& +\frac{1}{2}\left(I_{1,-1}-\exp \{\mathrm{i} 2 \phi\} I_{-1,-1}\right) \\
& +\frac{1}{2 \sqrt{2}}\left(\exp \{-\mathrm{i} \phi\} I_{2,0}-\exp \{\mathrm{i} 3 \phi\} I_{-2,0}\right),
\end{aligned}
$$

A Carr-Purcell echo

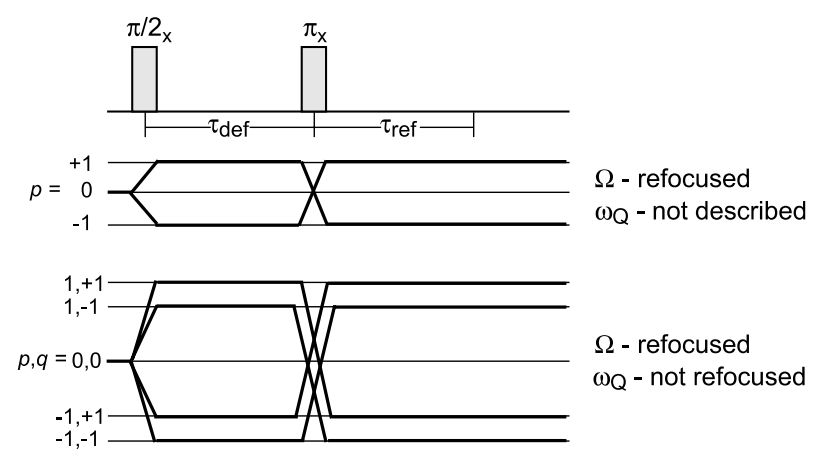

B Quadrupolar echo with Exorcycle

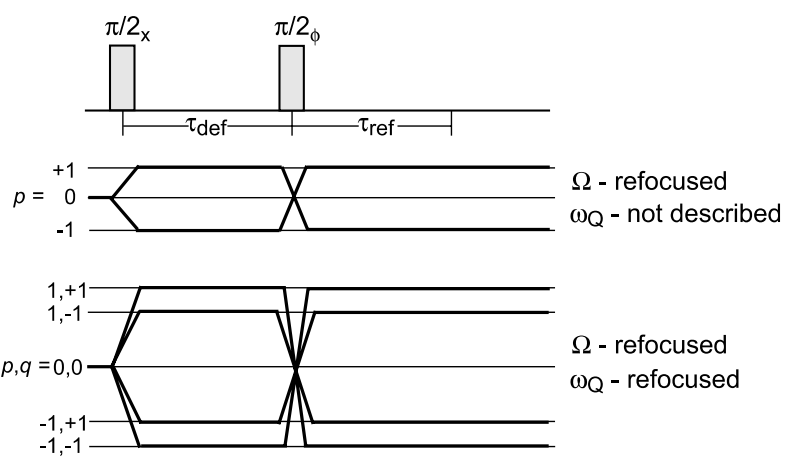

C Quadrupolar echo with anti-Exorcycle
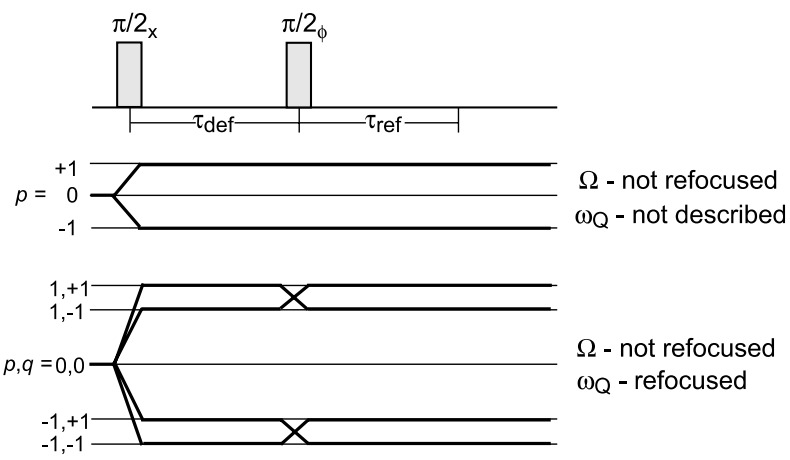

Fig. 4. (A) Zeeman echo induced by a $\pi$ refocusing pulse, (B) quadrupolar echo with a $\pi / 2$ refocusing pulse combined with the Exorcycle phase cycling scheme, and (C) quadrupolar echo, also with a $\pi / 2$ refocusing pulse, but used with the anti-Exorcycle scheme. The conventional coherence transfer pathways only indicate the coherence order $p$ and do not give any insight into the formation of quadrupolar echoes. The quadrupolar transfer pathways drawn for $I=1$ also indicate the satellite order $q$ and allow one to appreciate the difference between quadrupolar echoes, where first-order quadrupolar interactions $\omega_{\mathrm{Q}}$ are refocused, as in sequence (B) and (C), and Zeeman echoes, where chemical shifts and offsets $\Omega$ are refocused, as in (A and B).

$$
\begin{aligned}
I_{1,-1} \stackrel{(\pi / 2)_{\phi}}{\rightarrow} & -\frac{1}{2 \sqrt{2}} \exp \{\mathrm{i} \phi\}\left(I_{0,0}^{+1}-I_{0,0}^{-1}\right) \\
& +\frac{1}{2}\left(I_{1,+1}-\exp \{\mathrm{i} 2 \phi\} I_{-1,+1}\right) \\
& +\frac{1}{2 \sqrt{2}}\left(-\exp \{-\mathrm{i} \phi\} I_{2,0}+\exp \{\mathrm{i} 3 \phi\} I_{-2,0}\right)
\end{aligned}
$$




$$
\begin{aligned}
& I_{-1,-1} \stackrel{(\pi / 2)_{\phi}}{\rightarrow}-\frac{1}{2 \sqrt{2}} \exp \{-\mathrm{i} \phi\}\left(I_{0,0}^{+1}-I_{0,0}^{-1}\right) \\
&+\frac{1}{2}\left(I_{-1,+1}-\exp \{-\mathrm{i} 2 \phi\} I_{1,+1}\right) \\
&+\frac{1}{2 \sqrt{2}}\left(-\exp \{-\mathrm{i} 3 \phi\} I_{2,0}+\exp \{\mathrm{i} \phi\} I_{-2,0}\right) \\
& I_{-1,+1} \stackrel{(\pi / 2)_{\phi}}{\longrightarrow}-\frac{1}{2 \sqrt{2}} \exp \{-\mathrm{i} \phi\}\left(I_{0,0}^{+1}-I_{0,0}^{-1}\right) \\
&+\frac{1}{2}\left(I_{-1,-1}-\exp \{-\mathrm{i} 2 \phi\} I_{1,-1}\right) \\
&+\frac{1}{2 \sqrt{2}}\left(\exp \{-\mathrm{i} 3 \phi\} I_{2,0}-\exp \{\mathrm{i} \phi\} I_{-2,0}\right)
\end{aligned}
$$

Note that the imaginary coefficients of the polarization operators $I_{0,0}^{r}$ cancel if the starting density operator is Hermitian, e.g., if $\sigma(0)=I_{1,+1}+I_{-1,-1}$. A generalization of Eqs. (7)-(10) to spins $I>1$ is straightforward but of limited use, since such systems usually have large quadrupolar interactions making it difficult to achieve non-selective rotations.

The "Exorcycle" phase-cycling scheme [19] can be used to select operators that undergo a change of the coherence order $\Delta p=p^{\prime}-p= \pm 2$. This consists in repeating the experiment four times while incrementing the phase $\phi$ of the second pulse in steps of $\pi / 2$, combined with alternating addition and subtraction of the spectra

$I_{1,+1} \stackrel{(\pi / 2)_{\phi}}{\rightarrow} \frac{1}{2} I_{-1,-1}$ (all other terms being eliminated by

$$
\text { Exorcycle). }
$$

In this case illustrated in Fig. 4B, the change in sign $p^{\prime}=-p$ is accompanied by a change in sign $q^{\prime}=-q$, so that both Zeeman and first-order quadrupolar effects are refocused. This is particularly useful when recording deuterium spectra of paramagnetic solids [10]. Note the factor 1/ 2 in Eq. (11): half the signal intensity is lost compared to a conventional quadrupolar echo where the refocusing pulse is applied with a phase shift of $\pi / 2$ with respect to the first pulse. This is the price that must be paid for simultaneous refocusing of both $\Omega$ and $\omega_{\mathrm{Q}}$. Alternatively, an "anti-Exorcycle" scheme can be used (Fig. 4C) to retain only signals arising from operators that retain their coherence order $p^{\prime}=p$. This can be achieved by adding four spectra obtained while incrementing $\phi$ in steps of $\pi / 2$

$I_{1,+1} \stackrel{(\pi / 2)_{\phi}}{\rightarrow} \frac{1}{2} I_{1,-1}$ (all other terms being eliminated by anti-Exorcycle).

In this case, the offset $\Omega$ is preserved ( $p^{\prime}=p$ ) while the firstorder quadrupolar interaction is refocused $\left(q^{\prime}=-q\right)$ with a scaling of the signal by a factor $1 / 2$. Once again, this feature of the experiment can be readily appreciated by inspection of the quadrupolar transfer pathways.

The idea of retaining the shift information while refocusing the first-order quadrupolar interaction has been implemented in recent two-dimensional correlation experi-
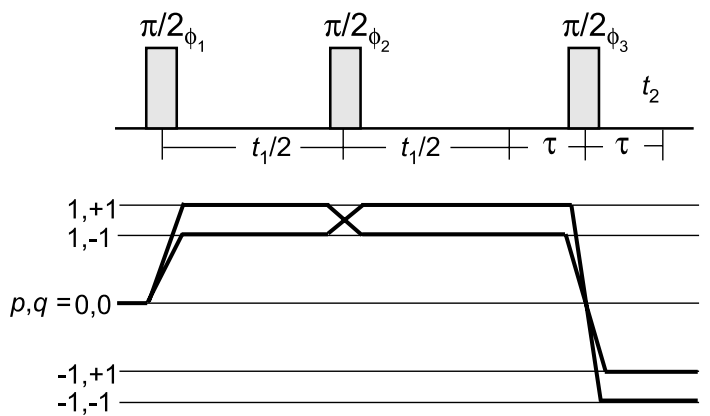

Fig. 5. Pulse sequence and quadrupolar transfer pathways for a twodimensional correlation method designed to separate quadrupolar $\omega_{\mathrm{Q}}$ and chemical shift anisotropy $\Omega$ interactions for $I=1$. The experiment refocuses $\omega_{\mathrm{Q}}$ but not $\Omega$ in the evolution period $t_{1}$, and refocuses both $\Omega$ and $\omega_{\mathrm{Q}}$ over the two $\tau$ periods. These effects are readily understood by inspection of the quadrupolar transfer pathways.

ments designed to separate these two interactions [20]. An appropriate pulse sequence is shown in Fig. 5 along with the quadrupolar pathway diagram. At the end of the $t_{1}$ evolution period, only the offset has lead to a net evolution while the first-order quadrupolar interaction is refocused. The duration of the subsequent $\tau$ periods is chosen so that one can observe the full echo in the $t_{2}$ detection period. This second echo is formed in such a way that there is no evolution under either offset or first-order quadrupolar interaction over the $2 \tau$ period, as can again be seen from the quadrupolar transfer pathways.

Similar refocusing effects can be observed in dipolarcoupled systems with two equivalent spins $I=1 / 2$ as occur in isolated water molecules in solids such as $\mathrm{CaSO}_{4} \cdot 2 \mathrm{H}_{2} \mathrm{O}$ [21]. In this case, one may speak of dipolar echoes, which obey the same rules as quadrupolar echoes. In systems with scalar couplings between two inequivalent nuclei $I=1 / 2$ and $S=1 / 2$ as occur in liquids, the offsets (chemical shifts) can be refocused by converting $|\alpha\rangle\langle\beta|$ (with $p=+1$ ) into $|\beta\rangle\langle\alpha|$ (with $p=-1$ ). If one applies $\pi$ refocusing pulses to a two-spin system, this leads to coherence transfer processes such as $|\alpha \alpha\rangle\langle\alpha \beta|\rightarrow| \beta \beta\rangle\langle\beta \alpha|$, leading to echoes that are modulated by the homonuclear coupling $J_{\text {IS. }}$. On the other hand, refocusing pulses with $\beta \neq \pi$ may lead to coherence transfer processes such as $|\alpha \alpha\rangle\langle\alpha \beta|\rightarrow| \alpha \beta\rangle\langle\alpha \alpha|$, leading to a refocusing of the homonuclear coupling. Perfect refocusing of both offsets and scalar interactions (or homonuclear dipolar couplings in solids) can be achieved with a sequence $(\pi / 2)_{x}-\tau-(\pi)_{x}-\tau-(\pi / 2)_{y}-\tau-(\pi)_{x}-\tau$-echo.

Quadrupolar transfer pathways are also useful to describe experiments designed for half-integer quadrupolar nuclei. These often exhibit large quadrupolar interactions so that second-order terms should be taken into account. The central transitions $(q=0)$ in single- or multiple-quantum spectra are only affected by the second-order quadrupolar interaction, while the satellite transitions $(q \neq 0)$ in single- and multiple-quantum spectra are affected both by first- and second-order quadrupolar interactions. Under magic angle spinning (MAS), zeroth-rank (isotropic) 
interactions such as the offset $\Omega$ remain unaffected. The first-order quadrupolar interaction and the second-rank part of the second-order quadrupolar interaction are completely averaged out. However, the fourth-rank part of the second-order quadrupolar interaction is merely scaled. Two-dimensional satellite-transition MAS (STMAS) experiments $[22,23]$. allow one to correlate the fourth-rank part of the second-order quadrupolar interaction of the satellite transitions $(q \neq 0)$ in the $t_{1}$ evolution period with the central transition $(q=0)$ in the $t_{2}$ detection period. It is possible to obtain high-resolution isotropic spectra by skew projections. From the quadrupolar pathway diagram for the shifted-echo STMAS [24] drawn for a spin $I=3 / 2$ in Fig. 6A it is evident that all three SQC coherences $I_{1, q}$ evolve during the evolution period $t_{1}$. Other coherences with $p \neq+1$ are readily filtered out by an appropriate phase cycling scheme. However, the desired satellites $q= \pm 2$ (drawn with solid lines) cannot be easily separated from the central transition $q=0$ (drawn with a dashed line). As a consequence the two-dimensional STMAS spectra show not only the desired correlation between the two satellite transitions $(q= \pm 2)$ in $t_{1}$ and the central transition $(q=0)$ in $t_{2}\left(I_{1, \pm 2} \rightarrow I_{-1,0}\right)$, but they also feature undesirable signals due to autocorrelation of the central transition coherence which persists throughout the $t_{1}$ and $t_{2}$ periods $\left(I_{1,0} \rightarrow I_{-1,0}\right)$. For nuclei with $I>3 / 2$, additional correlation peaks can be observed, such as signals that result from the correlation of outer satellites $(|q|>2)$ with the central transition $(q=0)$. No phase cycling method can filter out such unwanted correlations.

The double-quantum filtered DQF-STMAS experiment drawn for a spin $I=3 / 2$ in Fig. 6B utilizes a brief interval $\tau_{\mathrm{DQ}}$ where double-quantum coherences are excited to cancel unwanted autocorrelation $I_{1,0} \rightarrow I_{-1,0}$ signals [18,24]. From the quadrupolar pathways it is evident that the desired first-satellite coherences $I_{1, \pm 2}$ (solid lines in Fig. 6B) evolve in the $t_{1}$ period until they are converted into double-quantum coherences $I_{2, \pm 2}$ using a selective $\pi$ pulse applied to the central transition. At the same time the central transition coherence $I_{1,0}$ will be converted into $I_{-1,0}$ (dashed line in Fig. 6B). Here, phase cycling can be used to select coherences involving a change of the coherence order $\Delta p=p^{\prime}-p=+1$ (solid lines) while filtering out the unwanted coherence transfer process with $\Delta p=-2$ (dashed line). Similarly, for half-integer spins with $I>3 / 2$ (as illustrated for $I=5 / 2$ in Fig. 7), the outer-satellite coherences $|q|>2$ that evolve in the $t_{1}$ period can be filtered out as they retain their coherence order $\Delta p=p^{\prime}-p=0$ during the selective $\pi$ pulse applied to the central transition.

A final example of quadrupolar transfer pathways is shown for the SCAM-STMAS experiment that is self-compensated for angle misset $[25,26]$. Complete averaging of the first-order quadrupolar interaction can only be achieved if the magic angle is adjusted very accurately; otherwise the resolution in the isotropic dimension is limited by the residual first-order quadrupolar interaction.
A

STMAS
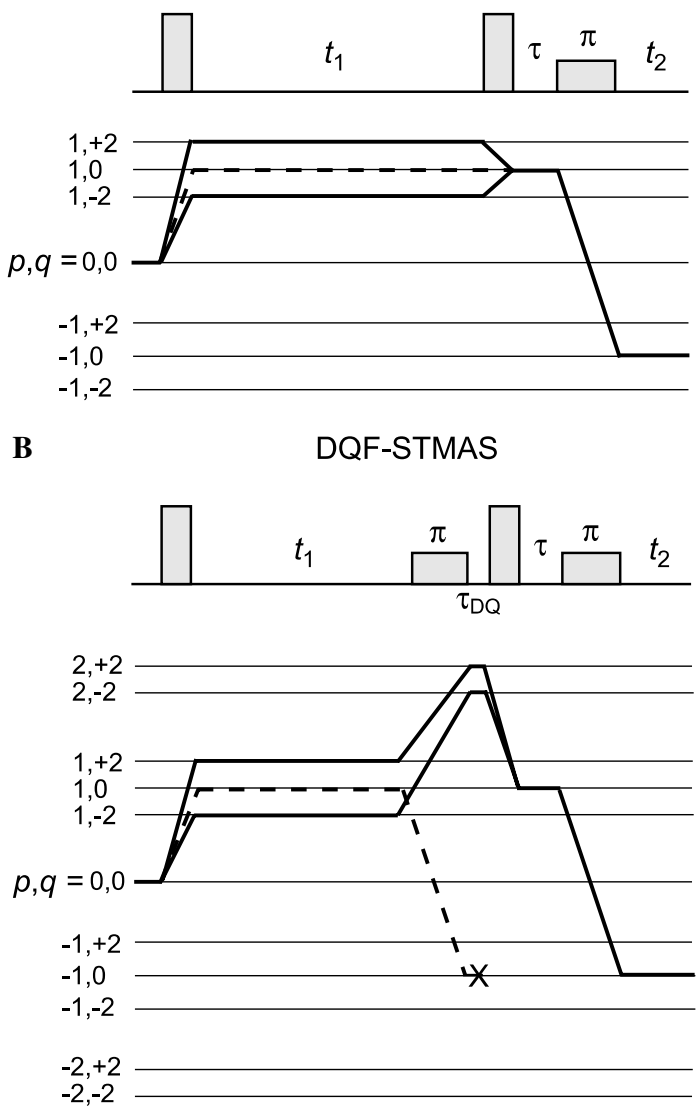

C

SCAM-STMAS

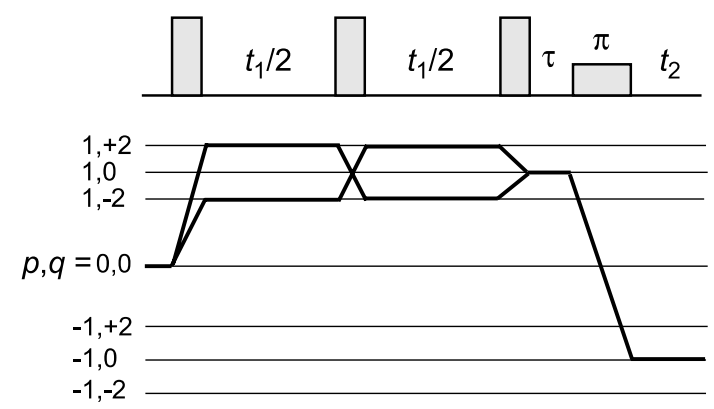

Fig. 6. Pulse sequences and quadrupolar transfer pathways for spin $I=3 / 2$ for (A) STMAS, (B) DQF-STMAS, and (C) SCAM-STMAS. The basic ideas of these experiments which are designed to obtain highresolution spectra of half-integer quadrupolar nuclei are readily understood by distinguishing the satellite order $q$ in the quadrupolar transfer pathways. The solid lines represent desired pathways for arbitrary spin-half quadrupolar nuclei, while parasitic pathways are drawn with dashed lines.

The SCAM-STMAS experiment has been proposed to overcome this problem. It differs from the STMAS experiment in Fig. 6A by addition of a pulse applied in the middle of the $t_{1}$ evolution period as illustrated in Fig 6C. Ideally, this pulse interconverts the single-quantum satellite coherences $I_{1, \pm 2} \rightarrow I_{1, \mp 2}$, while phase cycling can be used to suppress coherences undergoing a change in coherence order $I_{p, q} \rightarrow I_{p^{\prime}, q^{\prime}}$ where $p \neq p^{\prime}$. Unfortunately, parasitic processes such as $I_{1, \pm 2} \rightarrow I_{1, \pm 2}, I_{1,0} \rightarrow I_{1, \pm 2}$, and $I_{1, \pm 2} \rightarrow I_{1,0}$ that are 
DQF-STMAS

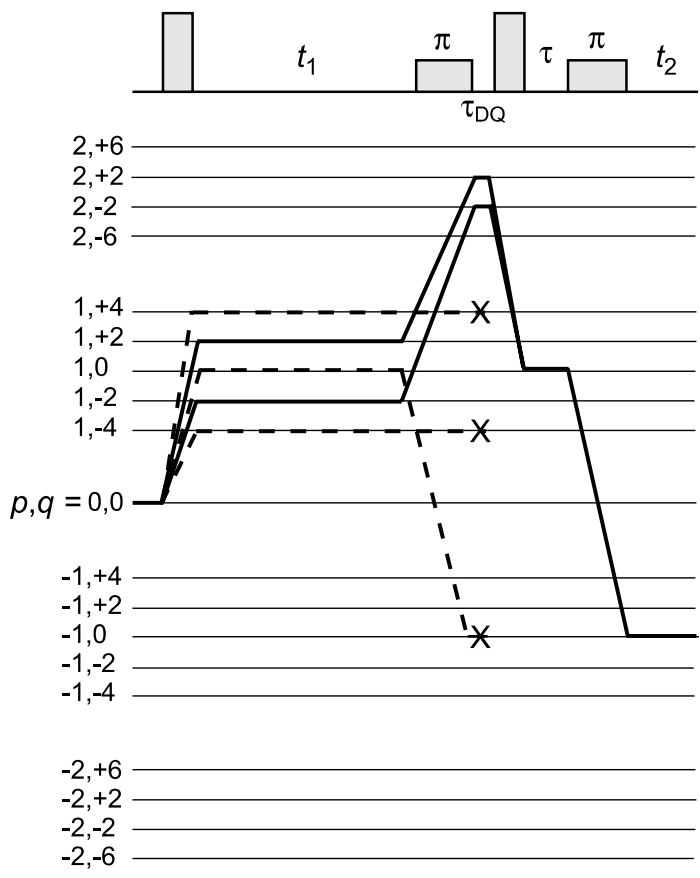

Fig. 7. Pulse sequences and quadrupolar transfer pathways for DQFSTMAS for spin $I=5 / 2$, analogous to Fig. 6 B.

not shown in Fig. 6C cannot be cancelled, though the latter two could probably be eliminated in a two-fold doublequantum filtered experiment that we might tentatively call DQF-SCAM-DQF-STMAS. The success of such experiments depends on the amplitudes of various coherence transfer processes, i.e., on parameters that cannot be predicted by pathways alone. The evolution of the desired coherences under the first-order quadrupolar interaction resulting from magic angle misset is eliminated through the formation of a quadrupolar echo at the end of the $t_{1}$ period, hence leading to a high-resolution isotropic spectrum even if there is a deviation from the magic angle. The main idea of SCAM-STMAS experiments may be appreciated by inspection of the quadrupolar transfer pathways.

\section{Conclusions}

To summarize, an extension of conventional coherence transfer pathways allows one to describe various experiments designed for quadrupolar nuclei in solids. Singleelement operators are classified according to their coherence order $p$ and their satellite order $q$. The objective of this paper is not merely to describe known experiments, but the hope that our graphical conventions may inspire further discoveries in this lively area of research.

\section{Acknowledgments}

We are indebted to Steve Wimperis, Zhehong Gan, Christian Fernandez, Dominique Massiot, and Philip
Grandinetti for constructive criticism. This work has been supported by the Fonds National de la Recherche Scientifique (FNRS, Switzerland), the Commission pour la Technologie et l'Innovation (CTI, Switzerland), and the Centre National de la Recherche Scientifique (CNRS, France).

\section{References}

[1] A.D. Bain, Coherence levels and coherence pathways in NMR. A simple way to design phase cycling procedures, J. Magn. Reson. 56 (1984) 418-427.

[2] G. Bodenhausen, H. Kogler, R.R. Ernst, Selection of coherencetransfer pathways in NMR pulse experiments, J. Magn. Reson. 58 (1984) 370-388.

[3] R.R. Ernst, G. Bodenhausen, A. Wokaun, Principles of Nuclear Magnetic Resonance in One and Two Dimensions, Oxford University Press, Oxford, 1987.

[4] P.J. Hore, J.A. Jones, S. Wimperis, NMR: The Toolkit, Oxford University Press, Oxford, 2000.

[5] J. Keeler, Understanding NMR Spectroscopy, Wiley, London, 2005.

[6] E.L. Hahn, Spin echoes, Phys. Rev. 80 (1950) 580-594.

[7] H.Y. Carr, E.M. Purcell, Effects of diffusion on free precession in nuclear magnetic resonance experiments, Phys. Rev. 94 (1954) 630-638.

[8] I. Solomon, Multiple echoes in solids, Phys. Rev. 110 (1958) 61-65.

[9] J.H. Davis, K.R. Jeffrey, M. Bloom, M.I. Valic, T.P. Higgs, Quadrupolar echo deuteron magnetic resonance spectroscopy in ordered hydrocarbon chains, Chem. Phys. Lett. 42 (1976) 390-394.

[10] S. Antonijevic, S. Wimperis, Refocussing of chemical and paramagnetic shift anisotropies in ${ }^{2} \mathrm{H}$ NMR using the quadrupolar-echo experiment, J. Magn. Reson. 164 (2003) 343-350.

[11] A. Wokaun, R.R. Ernst, The use of multiple quantum transitions for relaxation studies in coupled spin systems, Mol. Phys. 36 (1978) $317-341$.

[12] G. Jaccard, S. Wimperis, G. Bodenhausen, Multiple-quantum NMR spectroscopy of $S=3 / 2$ spins in isotropic phase: a new probe for multiexponential relaxation, J. Chem. Phys. 85 (1986) 6282-6293.

[13] N. Müeller, G. Bodenhausen, R.R. Ernst, Relaxation-induced violations of coherence transfer selection rules in nuclear magnetic resonance, J. Magn. Reson. 75 (1987) 234-297.

[14] M. Mehring, V.A. Weberruss, Object-Oriented Magnetic Resonance, Academic Press, London, 2001.

[15] E. Wigner, Gruppentheorie, Friedrich Vieweg und Sohn, Braunschweig, 1931.

[16] A. Wokaun, R.R. Ernst, Selective excitation and detection in multilevel spin systems: application of single transition operators, J. Chem. Phys. 67 (1977) 1752-1758.

[17] S. Vega, Fictitious spin 1/2 operator formalism for multiple quantum NMR, J. Chem. Phys. 68 (1978) 5518-5527.

[18] H.-T. Kwak, Z. Gan, Double-quantum filtered STMAS, J. Magn. Reson. 164 (2003) 369-372.

[19] G. Bodenhausen, R. Freeman, D.L. Turner, Suppression of artifacts in two-dimensional J spectroscopy, J. Magn. Reson. 27 (1977) 511-514.

[20] S. Antonijevic, S. Wimperis, Separation of quadrupolar and chemical/paramagnetic shift interactions in two-dimensional ${ }^{2} \mathrm{H}(I=1)$ nuclear magnetic resonance spectroscopy, J. Chem. Phys. 122 (2005) 044312 .

[21] J.G. Powles, P. Mansfield, Double-pulse nuclear-resonance transients in solids, Phys. Lett. 2 (1962) 58-59.

[22] Z. Gan, Isotropic NMR spectra of half-integer quadrupolar nuclei using satellite transitions and magic-angle spinning, J. Am. Chem. Soc. 122 (2000) 3242-3243.

[23] Z. Gan, Satellite transition magic-angle spinning nuclear magnetic resonance spectroscopy of half-integer quadrupolar nuclei, J. Chem. Phys. 114 (2001) 10845-10853. 
[24] S.E. Ashbrook, S. Wimperis, High-resolution NMR of quadrupolar nuclei in solids: the satellite-transition magic angle spinning (STMAS) experiment, Prog. NMR Spectrosc. 45 (2004) 53-108.

[25] S.E. Ashbrook, S. Wimperis, High-resolution NMR spectroscopy of quadrupolar nuclei in solids: satellite-transition MAS with self- compensation for magic-angle misset, J. Am. Chem. Soc. 124 (2002) 11602-11603.

[26] S.E. Ashbrook, S. Wimperis, SCAM-STMAS: satellite-transition MAS NMR of quadrupolar nuclei with self-compensation for magicangle misset, J. Magn. Reson. 162 (2003) 402-416. 